\title{
Post-harvest development of anthracnose in pepper (Capsicum spp): etiology and management strategies
}

\begin{abstract}
Chilli anthracnose, caused by Colletotrichum spp, is one of the main causes for post-harvest decay of chilli. It can develop on the field, during long distant transport, cold storage and shelf-life. In conventional agriculture, the whole plant including the fruits, are sprayed with fungicides as a prerequisite for post-harvest control of chilli anthracnose. Due to consumer concerns regarding the use of synthetic fungicides and the demand for safer storage methods, the use of synthetic fungicides is no longer allowed for the post-harvest control of chilli anthracnose. As a result, studies on alternative methods to control post-harvest decay have been developed over the years along with the demand for safer storage methods. In this review, results published within the last decade have been summarized and alternative approaches to synthetic fungicides for post-harvest control of chilli anthracnose were discussed in detail. Overall, the use of natural antimicrobials, biocontrol agents, resistant cultivars and ozone shows promise as treatments that can be adopted on a commercial scale to control post-harvest chilli anthracnose caused by Colletotrichum species.
\end{abstract}

Keyword: Anthracnose; Chilli; Post-harvest management; Natural products; Colletotrichum spp 